Aventin C. (à paraître 2007). « Les arts de la rue pour observer, comprendre et aménager l'espace public ». TIGR (Travaux de l'Institut de Géographie de Reims), n ${ }^{\circ} 123$, dossier « Art et espace », Reims : Département de géographie, Université de Reims Champagne-Ardenne.

\title{
Les arts de la rue pour observer, comprendre et aménager l'espace public
}

Catherine Aventin

Les arts de la rue sont communément représentés comme des événements divertissants, apportant de l'animation dans les espaces publics, faisant souffler un petit air festif dans l'ordinaire urbain. Une fois dépassée cette première impression de légèreté, ils sont souvent perçues comme des interventions aux messages volontiers subversifs ou tout au moins critiques et provocateurs vis-à-vis de la société contemporaine. Occupant une place de plus en plus importante et visible dans les villes depuis une trentaine d'années, les arts de la rue ont donné lieu à de multiples réflexions. Mais l'on a surtout réfléchi sur la force politique, le poids symbolique ou encore économique attribués aux arts de la rue ou ressentis comme tel et peu se sont interrogés sur les rapports à l'espace public urbain considéré dans ses dimensions construites (le bâti), sensibles (ce qu'on perçoit par les différents sens) et sociales (les pratiques et les usages). La composante spatiale de ce domaine du spectacle vivant a souvent été « oubliée », négligée par la critique mais aussi par la recherche, alors qu'elle nous parait pouvoir être transversale aussi bien à l'étude de l'espace public et de ses ambiances, qu'à l'approche des arts de la rue. Entre autres parce que ces derniers nous semblent être un biais intéressant pour interroger l'événement et l'ordinaire de la ville.

En tant qu'architecte nous nous intéressons à l'espace dans sa complexité et nous considérons l'architecture bien plus qu'un ensemble de formes ou « d'objets » répondant à des fonctions. Aussi nous sommes particulièrement sensible au travail des artistes de rue : les architectes, comme les artistes, ne sont-ils pas des créateurs intervenant dans et pour un objet commun, c'est-à-dire l'espace ? A la fois forme construite mais aussi habitée de multiples façons, l'espace urbain est un des points communs à ces deux professions et nous pensons que le regard et les pratiques des artistes sur la ville et tout ce que cet événement artistique provoque, peuvent intéresser les professionnels de l'aménagement urbain, du projet à la réalisation.

\section{Ville et arts de la rue : une approche spatiale}

Sur un plan très général, on peut relever que ces pratiques artistiques ont lieu « hors les murs », c'est-à-dire littéralement en extérieur, dans la ville et son espace public. La plupart du temps en dehors, elles s'inscrivent également hors de l'institution et s'offrent souvent gratuitement, car ayant la volonté de s'adresser le plus facilement et le plus directement à un public fréquentant peu, d'ordinaire, les « lieux de l'art ». Ces pratiques sont fondamentalement plurielles et prennent des formes multiples, ce que traduit l'expression « arts de la rue » qui, sans entrer dans les débats autour des définitions et tentatives de classifications de ce genre artistique, englobe l'ensemble de ces pratiques diverses. Cette notion est souvent utilisée dans un sens proche d'autres expressions telles que « théâtre de 
Aventin C. (à paraître 2007). « Les arts de la rue pour observer, comprendre et aménager l'espace public ». TIGR (Travaux de l'Institut de Géographie de Reims), n ${ }^{\circ} 123$, dossier « Art et espace », Reims : Département de géographie, Université de Reims Champagne-Ardenne.

rue », « art urbain », « art public », « actions artistiques urbaines », etc. Dans cet article, nous utiliserons indifféremment les expressions « arts de la rue » ou « actions artistiques » pour désigner des œuvres (souvent théâtrales mais pas uniquement) où l'espace public urbain est la base même de la création ainsi que le lieu de représentation.

Il faut noter que bien que ce domaine de création artistique intéresse de plus en plus de monde (public comme institutions, étudiants, chercheurs), les travaux de recherche s'intéressant spécifiquement aux rapports spatiaux entretenus par les arts de la rue avec l'espace public urbain et ses usagers et réalisant une véritable analyse d'un point de vue spatial restent rares'.

Néanmoins, certaines études et recherches examinent les arts de la rue à travers les champs de l'artistique et de l'urbain. Elles considèrent en effet, comme l'écrit Philippe Chaudoir, qu' « en tout état de cause, ces pratiques diverses impliquent toutes une sorte de mise en public de la question de l'art en dehors de ses périmètres institutionnels classiques. Plus encore, c'est la plupart du temps d'espace urbain dont il est question (...) $\Downarrow^{2}$. Cependant, les liens entre les arts de la rue et l'espace urbain sont alors souvent abordés d'un point de vue politique et symbolique. Pour notre part, les actions artistiques, en s'inscrivant dans l'espace public, mettent alors en jeu des liens intersensoriels et envers autrui ${ }^{3}$.

\section{L'espace public comme espace construit, sensible et social}

Nous nous inscrivons dans la lignée des chercheurs pour qui l'espace public est un ensemble de dimensions construites (le bâti), sensibles (ce qu'on perçoit par les différents sens) et sociales (pratiques et usages des habitants, passants, commerçants, etc.). Aussi, l'espace public urbain n'est pas considéré comme immatériel ou virtuel. L'espace est défini comme le « creux de la ville » (à l'opposé du « plein » que constituent les constructions), c'est-à-dire, comme le désignent les professionnels tels que l'aménageur, l'architecte ou l'urbaniste, comme étant « les espaces ouverts, extérieurs au logement, complémentaires du bâti privé et public (rues, places, jardins publics, boulevards, passages, abords des ensembles d'habitation), opposés aux édifices publics (mairie, écoles, musée, théâtres, services publics...) et aux lieux publics de statut privé (cafés, cinémas, gares, centres

\footnotetext{
${ }^{1}$ Recherche bibliographique effectuée dans le cadre de notre thèse : AVENTIN Catherine, 2005, Les espaces publics à l'épreuve des actions artistiques, Nantes, Thèse de doctorat en sciences pour l'ingénieur, spécialité architecture, Université de Nantes, 414 p.

${ }^{2}$ CHHAUDOIR Philippe, 2004, « Arts de la rue et espace urbain », L'Observatoire des politiques culturelles, n²6.

${ }^{3}$ C'est notamment l'approche qui a été choisie par Jean-François Augoyard (philosophe et urbaniste) au laboratoire Cresson (Centre de Recherche sur l'Espace Sonore et l'environnement urbain, Ecole d'Architecture de Grenoble, associé au laboratoire nantais du CERMA (Centre de recherche méthodologique d'architecture) au sein de l'U.M.R. C.N.R.S. 1563 « Ambiances architecturales et urbaines »), mais également explorée par l'architecte Eric Monin (MONIN Eric, 2001, Ambiances et dispositifs éphémères en milieu urbain, Nantes, Thèse de doctorat en sciences pour l'ingénieur, spécialité architecture, Université de Nantes, 2 tomes : 375 p. et 303 p.).
} 
Aventin C. (à paraître 2007). « Les arts de la rue pour observer, comprendre et aménager l'espace public ». TIGR (Travaux de l'Institut de Géographie de Reims), n ${ }^{\circ} 123$, dossier « Art et espace », Reims : Département de géographie, Université de Reims Champagne-Ardenne.

commerciaux...) $»^{4}$. Mais ce $«$ creux » n'est pas vide, ni en terme de matériels, ni en terme de sens. L'espace public a une forme et des matériaux qui vont, par exemple, modeler la propagation de sons, «sonner » de façon propre. Il reçoit et génère des pratiques, des morceaux de vie (individuelle et collective) s'y déroulent, il vit le passage des saisons, des événements s'y produisent, etc. C'est dans ce contexte, situé géographiquement et non pas « virtuel » (c'est-à-dire sans histoire et/ou sans « consistance » matérielle), que se produisent des événements, en l'occurrence des créations artistiques qui nous intéressent tout particulièrement.

Les espaces publics ne sont pas que le réceptacle ou même la conséquence d'activités qui s'y déroulent, c'est-à-dire que l'espace urbain n'est ni « passif » ni seul « cadre » des actions. L'espace urbain est un espace connu, perçu et vécu par les citadins qui entretiennent là des rapports à l'espace construit matériel via leur corps, et entrent ainsi en rapport avec autrui. En effet, l'espace public urbain et son architecture, sa forme, etc., " ne sont pas que des objets $\|^{5}$ et leurs « qualités sensibles ne sont pas des états fermés sur eux-mêmes et indépendants de l'activité du sujet percevant, elles sollicitent des conduites motrices qui les font apparaître en retour. Autrement dit, sentir et se mouvoir constituent deux versants indissociables du monde ambiant $»^{6}$. Etudier ces espaces publics signifie donc étudier les interrelations entre les citadins, " sujets » tout à la fois percevants (par toutes les modalités sensorielles, selon leur expérience, etc.) et actifs, et les lieux en question. Ces liens peuvent être aussi bien physiques (recelant $«$ offres $»^{7}$ et potentialités d'usages et d'actions) que sociaux ${ }^{8}$ (faire face aux autres...). Il paraît alors essentiel de prendre appui sur différentes disciplines, telles que l'architecture, la sociologie ou encore la psychologie de la perception afin d'appréhender ces liens.

Ainsi, il nous a paru possible d'interroger les relations constitutives de l'espace public en nous intéressant aux actions artistiques que sont les arts de la rue, d'autant que ces créations sont de formes diverses (musicales, théâtrales, plastiques, etc.) ayant pour base même la matière urbaine, en interaction avec l'espace public (au sens large, comme on l'a vu, construit, physique et sensible) et avec les pratiques ordinaires des citadins. Ces spectacles se produisent comme autant d'événements (en l'occurrence artistiques) qui s'insinuent, perturbent, rompent

\footnotetext{
${ }^{4}$ Extrait d'un ouvrage collectif du Plan Urbain (France) portant sur le thème des espaces public, cité par DUCRET André, 1994, L'art dans l'espace public. Une analyse sociologique, Zurich, Seismo, p. 143.

${ }^{5}$ AUGOYARD Jean-François, 1995, «L'environnement sensible et les ambiances architecturales », L'espace géographique, $\mathrm{n}^{\circ} 4$, p. 316.

${ }^{6}$ THIBAUD Jean-Paul, 2002, «L'horizon des ambiances urbaines », Communication n ${ }^{\circ} 73$ (« Manières d'habiter »), p. 189.

${ }^{7}$ Nous prenons appui sur le concept d’affordance développé par James J. Gibson que nous élargissons : nous considérons que les « offrandes » d'un lieu ne sont pas uniquement attachées à l'objet (dans notre cas à l'espace, aux propriétés physiques de l'espace public ), mais aussi aux « dispositions » des citadins (humeur, recherche particulière, moment, etc.).

${ }^{8}$ Nous nous référons principalement aux études d'Erving Goffman sur les rites d'interactions, sur les relations en publics, où il met à jour des comportements, des gestes, inhérents à des situations de coprésence des individus dans l'espace public.
} 
l'ordinaire de la ville et de la vie qui s'y déroule. Il nous semble alors que les actions artistiques peuvent permettre d'appréhender l'ordinaire de la ville et de la vie qui s'y développe, en tant qu'événement perturbateur du quotidien familier, et peuvent rendre possible une perception, offrir une «prise de conscience » de l'existence et de la forme de cet ordinaire. Les spectacles de rue sont donc des biais intéressants car ils « bousculent» l'habituel et activent les capacités d'adaptation des citadins. Ces derniers sont conduits notamment à réagir rapidement face à une situation nouvelle et exceptionnelle, et ainsi, peutêtre, à avoir un autre usage de l'espace, adopter un autre comportement, au moins le temps de cet événement.

En travaillant dans et avec l'ordinaire de la ville, les artistes de compagnies de rue interviennent au cœur de l'ensemble des relations constitutives de l'espace public, que ce soit l'espace avec ses qualités physiques et sensibles, les habitants et usagers de ces lieux, les interrelations existantes. Ils se trouvent en quelque sorte dans une situation transversale aux composantes de l'espace urbain, ce qui permet d'autant mieux l'étude de ce type d'espace et ce qui s'y déroule, dans toute leur complexité.

Par ailleurs, si les actions artistiques font ressortir les compétences ordinaires des habitants ou des passants, elles mettent aussi en lumière les compétences propres aux artistes, plus spécifiquement spatiales, qui font partie de la réalité quotidienne de leur travail. Bien que leur perception de la ville soit singulière (de par leur formation, leur expérience, leurs objectifs, etc.), leurs interventions, de par la nature même de leur cadre, peuvent être rapportées à celles des architectes et urbanistes, comme nous le verrons dans la suite de cet article.

\section{De l'événement à l'ordinaire de la ville}

\section{De l'événement}

La vie urbaine est peuplée d'événements de tous ordres qui interrompent le quotidien', qui viennent « perturber » l'espace et son activité quotidienne, en modifier le rythme, les pratiques, etc. L'événement crée en quelque sorte un « arrachement aux perceptions et représentations routinières $»^{10}$, déstabilise l'expérience du quotidien, du vécu ordinaire.

Ces événements étranges, c'est-à-dire sortant de l'ordinaire, peuvent faire apparaître différemment l'espace et ce qui peut s'y produire. Pour Henry Maldiney, « dans le sentir, un événement se fait jour à notre propre jour, lequel ne se lève qu'avec lui. Ne nous y trompons

\footnotetext{
${ }^{9}$ Par exemple des travaux qui obligent à changer de trottoirs et ainsi modifier un trajet quotidien, un accident de la circulation qui fait ralentir le pas, un bruit inaccoutumé qui fait aller à la fenêtre pour voir ce qui se passe, etc.

${ }^{10}$ AUGOYARD Jean-François, 1994, Actions artistiques en milieu urbain, A l'écoute d'une épiphanie sonore. Etude d'accompagnement de l'action sur l'environnement sonore urbain de Nicolas Frize à Saint Denis entre 1991 et 1993. Grenoble, Cresson - Plan Urbain, Ministère de l'équipement, du transport et du tourisme, p. 51.
} 
pas : un événement ne se produit pas dans le monde ; c'est lui au contraire, qui ouvre le monde en se produisant, et nous donne ouverture au monde et à nous-mêmes. Avec l'événement, s'ouvre la dimension de l'existence. Exister est avoir sa tenue hors... hors de toute contenance, à l'avant de soi. Tout événement est transformateur. Chacun vit en lui une transformation de sa présence comme être au monde $»^{11}$. La perception du monde qui nous entoure s'en trouve renouvelée, nous faisant en quelque sorte « renaître » au monde. Pour le chercheur également, ces événements vont créer des failles, des « brèches $»^{12}$, autrement dit mettre à jour des écarts de sens qui nous questionnent sur notre environnement et sur les relations sociales qui s'y développent. Nous pensons qu'ils peuvent rendre ainsi accessible au chercheur non seulement l'extraordinaire, mais aussi l'espace et le temps ordinaires de la ville.

\section{De l'ordinaire}

Comme nous venons de le voir, l'exceptionnel peut interrompre ou perturber l'ordinaire, le banal, autrement dit, ce qui «se définit d'abord négativement [comme] n'étant pas l'étrange $»^{13}$. Cet ordinaire, c'est ce que nous ne voyons plus, « ce qui semble avoir cessé à jamais de nous étonner $\gg^{14}$ et qui est la composante essentielle du quotidien ${ }^{15}$ et qui forme «l'infra-ordinaire, le bruit de fond $»^{16}$ habituel de la vie. Cet ordinaire de l'espace public, c'est l'espace qui finit par disparaître aux yeux des citadins, comme à force d'être vu, et qui n'est plus regardé. C'est ce qui nous est familier, ce qui s'offre comme habituel, « l'implicite, le non-conscient, le routinier, le banal... $\gg^{17}$. On peut supposer que cet effacement vient certainement en partie du fait qu' " en tant que quotidien, l'ordinaire est un présent répétable chaque jour ${ }^{18}$. Si la répétition (de gestes, de trajets, de paroles échangées avec un voisin, etc.) peut participer à rendre « habitable $»^{19}$ l'espace public (puisque c'est celui-ci qui nous intéresse), elle tend aussi à le rendre « invisible ». Non seulement les espaces quotidiens

\footnotetext{
${ }^{11}$ MALDINEY Henri, 1990, «La vérité du sentir », Art Press, n¹53, p. 17.

${ }^{12}$ CHALAS Yves, 1984, TORGUE Henry et SANSOT Pierre (dir.) L'imaginaire technique ordinaire, C.N.R.S. Sciences, Technique et Société, ESU.

${ }^{13}$ SAMI-ALI Mahmoud, 1980, Le banal, Paris, NRF Gallimard, p. 195.

${ }^{14}$ PEREC Georges, 1989, L'infra-ordinaire, Paris, Le Seuil, p. 12.

${ }^{15}$ Cet ordinaire peut néanmoins être sujet à des variations, prises telles qu'au sens musical du terme (« variations sur un même thème »). Cela signifie que des modifications peuvent être apportées au rythme quotidien, aux habitudes, mais sans que ceux-là s'en trouvent fondamentalement changés.

${ }^{16}$ PEREC Georges, 1989, Op. cit., p. 11.

${ }^{17}$ Ibid.

${ }^{18}$ AUGOYARD Jean-François, 1979, Pas à pas, Paris, Le Seuil, p. 137.

${ }^{19}$ Comme le montrent et l'expliquent par exemple Michel de CERTEAU dans (1980) L'invention du quotidien, 1. arts de faire. Paris, Gallimard, 350 p., Jean-François AUGOYARD à travers les trajets quotidiens des habitants (AUGOYARD Jean-François, 1979, Op. cit.) ou encore Yves CHALAS dans (1988) « La routine. Analyse d'une composante de la vie quotidienne à travers les pratiques d'habiter » Cahiers Internationaux de Sociologie, n LXXXV, pp. 243-256.
} 
devenant (trop) ordinaires et qu'on ne voit plus, mais aussi les pratiques et les savoirs ordinaires dont on ne parle pas et qui ne sont généralement pas formalisés.

Les actions artistiques peuvent amener à un niveau de conscience manifeste aussi bien des caractéristiques de l'espace que des comportements qui s'y déroulent, dispositifs et usages qui sont donnés comme habituels, comme évident. Ainsi, comme en temps ordinaire, les citadins développent des stratégies et des logiques d'installation dans l'espace public ou encore gèrent les relations interpersonnelles. Les spectacles vont en plus exacerber ces comportements qui pourraient presque passer inaperçus ou tout au moins seraient beaucoup plus difficiles à faire émerger. Expériences déstabilisantes, les spectacles mettent en jeu des compétences ordinaires maitrisées par les citadins pour faire face à des situations nouvelles avec et dans l'espace public urbain. Experts de leur quotidien, les habitants et les usagers des lieux possèdent une expérience de multiples situations, une habileté à gérer l'exposition en public, les rapports à autrui, etc., dont ils n'ont généralement pas conscience. Par le biais des arts de la rue, nous avons donc pu relever un certains nombre d'attitudes et de façons de faire, liées à l'espace construit mais aussi social ${ }^{20}$.

\section{Des pratiques intéressant les professionnels de l'espace}

Par rapport aux « arts de faire » des artistes des arts de la rue, en tant qu'architecte, nous ne pouvons manquer de relever que les approches et la mise en œuvre de projets, urbains comme artistiques, sont voisines, bien que les buts et les façons de faire des artistes de rue et des architectes soient, dans le détail, différentes. Ainsi il est intéressant d'observer que des connaissances, des logiques de projet et leur mise en œuvre, pourrait être rapprochés les uns des autres, se parler et même s'enrichir.

\section{Des registres communs}

On peut noter chez les artistes du théâtre de rue, comme le relève déjà le sociologue Philippe Chaudoir, un certain $"$ parallélisme avec la pensée aménageuse $»^{21}$. Pour lui, cela se traduit dans le vocabulaire utilisé par les créateurs qui se rapproche de celui du champ de l'urbanisme (relative maitrise de termes ${ }^{22}$ et de filtres d'analyses), dans son registre ${ }^{23}$ comme dans l'attitude

\footnotetext{
${ }^{20}$ Pour les résultats, nous renvoyons à notre thèse.

${ }^{21}$ CHAUDOIR Philippe, 2000, Discours et figures de l'espace public à travers les arts de la rue, Paris, L'Harmattan, p. 231.

${ }^{22}$ Vocabulaire incluant également une série d'acronymes, de D.S.Q. (Développement Social des Quartiers) à G.P.V. (Grand Projet de Ville), au rythmes des programmes des politiques de la ville insufflés par les institutions.

${ }^{23}$ Philippe Chaudoir précise que « parler d'un parallélisme entre le discours des aménageurs et celui des artistes n'est peutêtre pas tout à fait la formulation adéquate. Il faudrait, pour être exact, se situer dans le registre de la comparaison à partir du seul propos des artistes. C'est donc en tout état de cause, en référence à un discours supposé des aménageurs que se fonde la comparativité ». in CHAUDOIR Philippe, 2000, Op. cit., p. 234.
} 
Aventin C. (à paraître 2007). « Les arts de la rue pour observer, comprendre et aménager l'espace public ». TIGR (Travaux de l'Institut de Géographie de Reims), n 123 , dossier « Art et espace », Reims : Département de géographie, Université de Reims Champagne-Ardenne.

générale d'analyse et de « diagnostic urbain ». Les artistes peuvent connaître et utiliser les codes de l'urbain et des professionnels qui y interviennent, mais aussi parfois entrer dans les programmes institutionnels ${ }^{24}$. Plus qu'un parallélisme de pensée, les deux professions se retrouvent également sur certaines questions qui sont renvoyées de l'un des groupes de professionnels à l'autre. Le politique demande souvent aux architectes et urbanistes de concevoir et proposer des espaces propres à une vie sociale, propres à la rencontre et où peut (voire, doit) se tisser du « lien social ». D’un autre côté, les aménageurs demandent aux artistes de jouer ce rôle d'animateurs et surtout « d'adjuvants du processus d'urbanisation »" Ce qui met ces créateurs d'autant plus en position de s'interroger sur la ville, sur l'espace public, au même titre que tout autre professionnel de l'espace.

\section{Logique de projet}

Que ce soit pour les artistes de rue ou pour les architectes et les urbanistes, se posent inévitablement les questions du contexte c'est-à-dire du moment et du lieu d'intervention, celui-ci possédant des qualités propres et des usages multiples qui varient selon des temps urbains. Les artistes auraient l'avantage de connaître par avance la dramaturgie qui va se jouer ${ }^{26}$ et auraient également une maîtrise plus importante sur le monde car ils créent euxmêmes des personnages qui investiront l'espace public. Pour autant, les artistes ne sont pas totalement libres puisqu'ils sont eux aussi confrontés à toutes sortes de contraintes : cela va des questions de budget à celles liées à la sécurité (des artistes comme des spectateurs), sans oublier les obligations réglementaires de tous ordres et les points techniques. Ainsi, les artistes, les architectes et les urbanistes ont à faire avec la ville, dans toutes ses composantes (physique, sensible, sociale, économique, réglementaire...). Du côté des architectes et des urbanistes, le risque est peut être que le concepteur voit les habitants et les usagers comme des êtres caricaturaux, désincarnés, voire abstraits mais à qui le projet serait destiné. Ce contexte commun fait d'ailleurs dire à Bernard Colin, de la compagnie Tuchenn : « Nous faisons le même travail que les architectes, nous avons le même matériau $»^{27}$. Il nous semble qu'outre ce « matériau » commun, les artistes et les architectes-urbanistes connaissent et manipulent des domaines multiples afin de donner forme et force au projet. Ils sont chacun tout à la fois artiste, technicien, ingénieur, etc.

\footnotetext{
${ }^{24}$ Comme ceux liés aux Développement Social Urbain (D.S.U.), Développement Social des Quartiers (D.S.Q.), etc.

${ }^{25} I d$., p. 68.

${ }^{26}$ Voir à ce sujet BOUCRIS Luc, 1993, «Les pratiques scénographiques aujourd'hui », Scénographie et espaces publics. Les lieux de la représentation dans la ville., Paris, Plan urbain, séminaire de recherche.

${ }^{27}$ Intervention lors d'un débat aux Rencontres professionnelles sur les arts de la rue organisées par HorsLesMurs à Paris (4 5 novembre 1998, La Villette). Notes personnelles.
} 


\section{Mise en auvre}

Concernant la réalisation du projet artistique, de sa préparation technique jusqu'à la présentation du spectacle, on se rend compte que le processus de mise en œuvre est souvent comparable à celui des projets urbains. En effet l'artiste, comme l'urbaniste ou l'architecte, est le maître d'œuvre et, de ce fait, doit rencontrer et faire travailler ensemble tout un ensemble d'acteurs très divers (simultanément ou successivement). Par exemple, dès la phase de repérage des lieux (c'est-à-dire en amont de la représentation), les artistes ont à leurs côtés un représentant du commanditaire mais également souvent une (ou des) personne(s) de services techniques municipaux qui sont les personnes au plus près du terrain et qui connaissent a priori le mieux tous les aspects techniques des espaces publics concernés ${ }^{28}$. Les demandes envers les services municipaux peuvent relever aussi bien du service voirie que de celui des espaces verts, et cela peut toucher également les questions de contrôle de « (...) l'accès des véhicules, les arrêtés municipaux d'interdiction de circulation et de stationnement, la sécurité des spectateurs, le nettoyage et la remise en état des sites $\${ }^{29}$, etc. L'artiste, comme l'architecte, se trouve coordinateur et interlocuteur privilégié des différents acteurs en jeu dans l'opération de projet.

\section{Des perspectives}

Malgré des objectifs et des arts de faire voisins mais différents, nous pensons que les professionnels de l'aménagement gagneraient à s'intéresser aux regards et aux pratiques des artistes de rue, à la fois pour percevoir, prendre conscience et comprendre au mieux l'espace public urbain, mais aussi imaginer et essayer des aménagements, tirer profit des potentialités d'un site. Ainsi, les actions artistiques qui se tiennent dans des lieux particuliers de la ville peuvent aussi être une façon de se rendre compte de ce que pourrait être cet endroit selon une autre configuration urbaine, avec d'autres usages, d'autres qualités mises en avant. Autrement dit, cela peut devenir une sorte de « test grandeur nature » pour un éventuel futur aménagement (par exemple des façades de bâtiments ou encore des constructions « simulées » grâce aux techniques des décors de théâtre, essais d'éclairage public concernant des dispositifs, des niveaux d'éclairement, des couleurs, etc.). Il est possible d'imaginer que les actions artistiques soient des moments permettant de se rendre compte de ce que seraient des sites avec une occupation par exemple uniquement piétonne ${ }^{30}$. La tenue d'actions artistiques éphémères dans la ville offre donc l'occasion de modifier formes, ambiances, usages,

\footnotetext{
${ }^{28}$ Suivant le site d'intervention d'autres acteurs peuvent être impliqués : Délégation Départementale à l'Equipement (D.D.E.), services de la préfecture, etc.

${ }^{29}$ ROBERT Jean, 2001, "Quand le théâtre investit les rues », La gazette des communes, des départements et des régions $\mathrm{n}^{\circ} 1560$, p. 20.

${ }^{30}$ Ainsi le détournement de la circulation automobile permet de tester d'autres itinéraires (et leurs conséquences) pour les voitures, aider à percevoir la nouvelle ambiance sonore ainsi générée sur le site quand il n'y a plus que des passants, de repérer peut-être déjà certains usages nouveaux par rapport aux pratiques habituelles, etc.
} 
Aventin C. (à paraître 2007). « Les arts de la rue pour observer, comprendre et aménager l'espace public ». TIGR (Travaux de l'Institut de Géographie de Reims), n ${ }^{\circ} 123$, dossier « Art et espace », Reims : Département de géographie, Université de Reims Champagne-Ardenne.

d'étudier l'espace et de se faire une première idée de ce que pourrait être un aménagement, une occupation autre, encore inédits et parfois insoupçonnés ${ }^{31}$.

Les espaces publics urbains étudiés par l'intermédiaire des arts de la rue et selon les dimensions construites, sensibles et sociales se donnent à observer et comprendre dans leurs moments non seulement extraordinaires (pendant les spectacles de rue) mais également ordinaires. En effet, les artistes de rue, par leurs interventions, redonnent à agir dans des espaces quotidiens, révélant mais aussi activant des potentialités d'actions, de perceptions, rendant plus « tangibles » la dimension charnelle et sensible des citadins eux-mêmes. A l'activation du corps se lie celle de l'espace matériel qui s'offre dans ses potentialités ordinaires, peu remarquables au quotidien, mais aussi plus extraordinaires, c'est-à-dire inconnues, rares ou inhabituelles, mais que l'on retrouve au cours de différents spectacles. Ces pratiques artistiques hors les murs sont donc une façon originale d'observer et de comprendre l'espace public urbain, et par là-même d'aider à repenser l'aménagement de ce dernier. Le lecteur aura compris qu'il ne s'agit pas de transformer la ville en théâtre et par conséquent de faire en sorte que l'espace public soit un grand plateau technique pour des interventions artistiques. Au contraire, nous pensons que les architectes et les urbanistes doivent proposer des aménagements qui, par leurs formes, leurs matériaux, leur mobilier urbain, etc., favorisent des usages multiples, les plus nombreux possibles, souvent même insoupçonnés. Ces aménagements doivent favoriser l'appropriation des lieux par tout un chacun, à tout moment et pouvant varier dans le temps. En fait, comme l'écrit justement Isaac Joseph, « mettre en scène l'espace public ce n'est donc pas l'apprêter pour un spectacle, faire qu'il en impose, c'est l'organiser sur un récit ou un parcours possible» ${ }^{32}$. C'est bien à des récits multiples que l'espace public doit pouvoir se prêter, ce sont des parcours forts différents les uns des autres qui doivent pouvoir se croiser, se superposer, coexister et que l'architecte et l'urbaniste doivent imaginer. En cela, il nous apparaît que la sensibilité, la connaissance et les façons de faire qu'ont les artistes de rue face à la ville, avec leurs créations originales, peuvent constituer un apport riche pour observer, comprendre et aménager l'espace public. Et notre travail montre que les artistes, comme le dit très bien Bruno Péquignot, " mettent à notre disposition des instruments supplémentaires, différents de ceux acquis auparavant, de notre perception, de reconnaissance, et donc de connaissance, de ce qui constitue notre expérience

\footnotetext{
${ }^{31}$ Ce qui peut intéresser tous les acteurs impliqués dans l'aménagement urbain, maîtres d'œuvres comme maîtres d'ouvrage.

${ }^{32}$ JOSEPH Isaac, 1992, « L'espace public comme lieu de l'action », Les Annales de la Recherche Urbaine, n57-58, p. 212.
} 
Aventin C. (à paraître 2007). «Les arts de la rue pour observer, comprendre et aménager l'espace public ». TIGR (Travaux de l'Institut de Géographie de Reims), n²123, dossier « Art et espace », Reims : Département de géographie, Université de Reims Champagne-Ardenne.

du monde ${ }^{33}$. Il nous semble donc que les créateurs des arts de la rue, par leurs regards et leurs interventions, pourraient permettre de stimuler l'imagination des professionnels du projet urbain et architectural pour inventer des possibles.

${ }^{33}$ PEQUIGNOT Bruno, «Introduction » in ANCEL Pascale, 1996, Une représentation sociale du temps. Etude pour une sociologie de l'art, Paris, L'Harmattan, p. 10. 